\title{
Evaluación de residuos de la industria forestal por un sistema de FSS de presacarificación con fines a la producción de alcohol
}

\author{
López, A. (1), Lopretti, M. (1), Tomasso, M. (2), Duarte, G. (3) \\ (1) Departamento de Bioprocesos y Biotecnología, Laboratorio Tecnológico del Uruguay, LATU - (2) Departamento de \\ Bioprocesos y Biotecnología (2007-2008), LATU - ${ }^{(3)}$ Departamento de Bioprocesos y Biotecnología (2007-2008), LATU \\ (febrero - mayo 2009) \\ Contacto: mlopre@latu.org.uy
}

Recibido: 2/7/2009 - Aprobado: 25/11/2009

\begin{abstract}
$\underline{\text { Resumen }}$
Con este trabajo se pretende colaborar en el desarrollo de la tecnología para la transformación de materiales lignocelulósicos en bioetanol mediante un proceso eficiente y económicamente rentable que facilite su implementación a nivel industrial. En un sistema de fermentación sólida se trataron residuos de la industria forestal mediante un procedimiento en dos etapas. Primero se inoculó el material con microorganismos que presentan actividad ligninolítica (Tramentes versicolor y Pleurotus ostreatus) y luego con microorganismos con actividad celulolítica (Trichoderma reesei).

Para evaluar los resultados se determinó el contenido de azúcares fermentables, donde pudo observarse que las muestras tratadas con hongos mostraron un mayor contenido al ser comparadas con muestras sin tratar. Otros parámetros evaluados fueron el ADF y el NDF, los cuales descendieron en el material tratado, manifestando una probable predigestión.

Se realizaron medidas de absorbancia a longitudes de onda entre $200 \mathrm{~nm}$ y $400 \mathrm{~nm}$ para evaluar los productos de degradación de lignina, observándose un aumento en las muestras tratadas. Para evaluar la efectividad del tratamiento se realizaron sacarificaciones enzimáticas sobre el material tratado y el material sin tratar, determinándose luego por DNS los azúcares reductores obtenidos. El resultado determinó que con el tratamiento se obtuvo un aumento en los azúcares del $45 \%$.

El aumento en los rendimientos obtenidos permite considerar su utilización como metodología de presacarificación de bajo costo. Se considera promisoria la aplicabilidad del proceso y debido a la variabilidad obtenida se seguirá trabajando en su optimización.

Palabras clave: residuos lignocelulósicos, microorganismos ligninolíticos, sacarificación enzimática, azúcares reductores.
\end{abstract}

\begin{abstract}
$\underline{\text { Abstract }}$
This work intends to collaborate in the development of the technology needed to transform lignocellulosic materials into bioethanol through a process that is both efficient and profitable so it can be deployed in the industry. In a system of solid fermentation, waste from the forestry industry was treated in a two stage process. At first, the material was inoculated with microorganisms that present ligninolytic activity (Tramentes versicolor y Pleurotus ostreatus) and then with microorganisms with cellulolytic activity (Trichoderma reesei). To assess the results, the fermentable sugars content was determined, and the samples treated with fungi showed a higher content than the untreated ones. Other parameters took into consideration where the ADF and the NDF, which were lower in the treated material, showing a plausible predigestion. Some measures of absorbance at wavelengths in between $200 \mathrm{~nm}$ and $400 \mathrm{~nm}$ were made to assess the products from the lignine degradation and it showed an increase in the treated samples. To assess the effectiveness of the treatment, enzymatic sacarifications were done on both treated and untreated material and the reductor sugars were later measured through DNS. As a result, an increase of $45 \%$ was found in the treated samples. The high performances obtained allows us to consider it as a low cost presacarification methodology. The applicability of the process appears to be promising and because of the high variability of the results there will be further work done in its optimization.

Keywords: lignocellulosic materials, ligninolytic microorganisms, enzymatic sacarifications, reductor sugars.
\end{abstract}

\section{Introducción}

La búsqueda de combustibles sustitutos del gasoil y la gasolina para lograr la autosuficiencia energética y la independencia de precios del petróleo se ha transformado en una meta para varios países que promueven el desarrollo industrial sustentable. Además, el calentamiento global de la atmósfera, la emisión de gases de efecto invernadero y las catástrofes ambientales producidas a partir de derrames de hidrocarburos han motivado aún más la exploración de fuentes de energía alternativas.

Dentro de este contexto aparecen como una gran oportunidad los biocombustibles, entre ellos el bioetanol, y entre las materias primas los residuos de la industria forestal como aportadores de celulosa y azúcares fermentables.

En contraste con lo que ocurre en la conversión de maíz a etanol, la ruta que involucra la conversión de celulosa a etanol implica poca o ninguna contribución a la emisión de gases de efecto invernadero (Demain, 2005).

La lignocelulosa es el componente principal de las plantas leñosas y no leñosas y representa la fuente principal de materia orgánica renovable. Grandes cantidades de "residuos" lignocelulósicos son generados a partir de las prácticas agrícolas y forestales que se 
transforman en un problema de polución ambiental. Lamentablemente, gran parte de los residuos lignocelulósicos se disponen para su quemado, lo cual no se restringe únicamente a los países en vías de desarrollo, sino que se considera un fenómeno global (Levine, 1996). Sin embargo, las grandes cantidades de biomasa residual de plantas consideradas como "desecho" podrían potencialmente convertirse en productos con valor agregado, incluyendo biocombustibles, químicos, fuentes de energía económica para fermentación, alimentación animal mejorada y nutrientes humanos

Los residuos lignocelulósicos están compuestos por lignina, hemicelulosa y celulosa. Debido a la complejidad de disolver la lignina sin destruirla, su estructura química exacta es difícil de dilucidar. La lignina está unida a la hemicelulosa y a la celulosa formando un sello físico alrededor de estos dos componentes, lo que genera una barrera impenetrable a soluciones y enzimas. Una de las problemáticas existentes es cómo deslignificar y predigerir la celulosa en forma eficiente y económica, lo cual luego permita hidrolizar y fermentar efectivamente.

De los tres componentes, la lignina es la más recalcitrante a la degradación, mientras que la celulosa es más resistente a la hidrólisis que la hemicelulosa. Tanto métodos de hidrólisis ácidos (Nguyen, 1993), como alcalinos (Chahal, 1992) han sido utilizados para degradar lignina. La optimización de pretratamientos químicos y físicos para fuentes específicas de biomasa es un campo de creciente interés (Ragauskas, 2006). Para muchos procesos las enzimas son preferidas a los procesos ácidos o alcalinos, ya que producen biocatálisis específicas, pueden operarse en condiciones menos extremas, no generan productos indeseados y son amigables con el medio ambiente.

Una herramienta importante para reducir el costo de esta depolimerización es el pretratamiento de estos lignocelulósicos para lograr una matriz biomásica más accesible a las enzimas. Los beneficios de estos pretratamientos están unidos a los recientes esfuerzos en investigación que han reducido los costos de las celulasas.

Uno de los pretratamientos biológicos utilizados es la fermentación en estado sólido (SSF). Otras estrategias para el bioprocesamiento de la lignocelulosa incluyen tratamientos anaeróbicos, composteo, producción de proteína unicelular para alimentación animal de rumiantes y el cultivo de hongos (Smith, 1987).

La fermentación en estado sólido se caracteriza por la completa o casi completa ausencia de líquido libre. El agua, esencial para la actividad microbiana, está presente en forma absorbida o complejada con la matriz sólida y el sustrato (Canel y Moo-Young, 1980). Estas condiciones de cultivo son adecuadas especialmente para el crecimiento de hongos, debido a que crecen en cantidades de agua relativamente bajas. Las SSF son prácticas para sustratos complejos que incluyen residuos agroindustriales, forestales y del procesamiento de alimentos y desechos utilizados como fuente de carbono para la producción de enzimas lignocelulolíticas.

El SSF es un proceso atractivo para producir enzimas microbianas utilizando materiales lignocelulósicos provenientes de desechos agroindustriales, debido al bajo capital de inversión y al bajo costo operativo (Chahal, 1996; Haltricht, 1996; Jecu, 2000). Este proceso, por lo tanto, sería ideal para los países en vías de desarrollo.

El objetivo primario de los tratamientos de los lignocelulósicos por las diferentes industrias es el acceso potencial a la celulosa incrustada en la matriz de lignocelulosa (Malherbe, 2003). Éstos establecen que la combinación de la tecnología de la SSF con la habilidad de un hongo apropiado para degradar lignina selectivamente hará posible la implementación a escala industrial de biotecnologías basadas en lignocelulosa.

Las especies del género Pleurotus presentan una serie de propiedades organolépticas y nutrimentales, así como una reconocida capacidad biodegradativa. Tienen capacidad de secretar diversas oxidasas y fenoloxidasas, lo que le permite crecer en sustratos que contienen lignina y compuestos fenólicos.

Los hongos de la pudrición blanca como Phanerochaete chrysosporium o Pleurotus ostreatus, pertenecientes a los basidiomicetes, y el hongo de podredumbre parda Gloeophyllum trabeum son los más eficientes degradadores de lignina (Gold y Alic 1993). La ruptura de lignina se produce a través del uso de una familia de enzimas extracelulares llamadas colectivamente "ligninasas". Dos familias de enzimas ligninolíticas juegan un rol clave en la degradación enzimática: fenol oxidasa (lacasa) y peroxidasa (Lignina peroxidasa) (LiP) y manganeso peroxidasa (MnP) (Krause, 2003; Malherbe y Cloete, 2003).

Estos hongos también producen enzimas extracelulares capaces de hidrolizar la celulosa. El complejo celulolítico incluye tres tipos de enzimas: la endo- $\beta-1,4$-glucanasa, la exo- $\beta$-1,4-glucanasa y la $\beta-1,4-$ glucosidasa. El hongo más estudiado a nivel piloto es Trichoderma reesei.

Las celulasas y las hemicelulasas tienen numerosas aplicaciones y potencial biotecnológico para la industria química, de combustibles, de alimentos, de bebidas, textil, pulpa y papel (Bhat, 2000; Sun y Cheng, 2002)

La reducción de costos en el bioprocesamiento se logrará por una combinación de los tratamientos que involucran pretratamientos y utilización de celulasas y hemicelulasa con otros pasos del proceso. Se ha propuesto una combinación en la producción de enzimas con los pasos de fermentación mediante microorganismos modificados capaces de producir celulasa y fermentación alcohólica, lo cual podría proveer de una liberación de la mezcla óptima de enzimas hidrolíticas específicas. En general, la fermentación de una mezcla de hexosas y pentosas es ineficiente, debido a que no se ha encontrado ningún organismo que pueda convertir todos los azúcares en etanol con altos rendimientos.

Durante más de 50 años una de las principales áreas de investigación de la biotecnología de lignocelulósicos ha sido conducida por la necesidad de aislar e identificar microorganismos que fueran productores de nuevas enzimas lignocelulolíticas. Desde la perspectiva de aplicación, el interés se ha focalizado además en encontrar enzimas que pudieran romper la lignocelulosa rápidamente, pero también que pudieran soportar $\mathrm{pH}$, temperatura y agentes inhibitorios más exitosos dependiendo de la intención de la aplicación.

Uno de los desafíos que plantea la biotecnología de lignocelulósicos es, por tanto, lograr la deslignificación a gran escala con objeto de liberar la celulosa para su utilización como fuente de azúcares fermentables. Los azúcares generados a partir de los lignocelulósicos son una mezcla de hexosas (glucosa) y pentosas (xilosa y arabinosa). Las hexosas pueden fácilmente ser fermentadas a etanol empleando cepas industriales de S. cerevisiae. Sin embargo, no se han encontrado variedades Saccharomyces que puedan fermentar pentosas como xilosas a etanol, ni otros organismos que combinen todas las características industriales relevantes requeridas para la fermentación efectiva de xilosa a etanol (Attfield y Bell, 2006).

Los pretratamientos de los lignocelulósicos permiten mejorar la reactividad de los polisacáridos, reducir el tiempo de hidrólisis (velocidad) y así aumentar el rendimiento (Mosier, 2006).

Actualmente están ocurriendo grandes avances en este sentido, por medio de los microorganismos genéticamente modificados. Otro desafío importante involucra la necesidad de entender y manipular la tolerancia a etanol y azúcares y la resistencia a potenciales inhibidores generados en los tratamientos de presacarificación.

\section{Materiales y Métodos}

\section{Preparación del sustrato}

De acuerdo a los residuos forestales disponibles se ensayaron diferentes mezclas para determinar aquella que permitiera un adecuado crecimiento fúngico incorporando el mayor contenido de aserrín posible. La mezcla seleccionada consistió en un $60 \%$ de chips de madera, $20 \%$ de aserrín y $20 \%$ de viruta.

Esta mezcla fue ensayada a dos escalas:

1. Escala banco: seis bandejas con $10 \mathrm{~K}$ cada una. Fueron rotuladas de 1 a 6 , la número 1 no fue inoculada inocular (blanco).

2. Escala campo: nueve cajones con $57 \mathrm{~K}$ cada uno. 


\section{Inoculación con hongos ligninolíticos}

1. Escala banco: Se inocularon cinco bandejas (rotuladas de 2 a 6 ) con $2 \mathrm{~K}$ de mezcla de partes iguales en peso de inóculo de Trametes versicolor y Pleurotus ostreatus crecido sobre semillas de trigo. Se preparó otra bandeja en las mismas condiciones pero sin inocular para que actuara como blanco (rotulada 1). Sesenta días más tarde se inocularon con Trichoderma reesei crecido sobre semillas de trigo con el fin de favorecer la sacarificación final (Figura 1).

2. Escala campo: Se inocularon los nueve cajones con $3 \mathrm{~K}$ de Trametes versicolor y $3 \mathrm{~K}$ de Pleurotus ostreatus crecido sobre semillas de trigo. Pasados los tres meses se inocularon con $3 \mathrm{~K}$ de Trichoderma reesei crecido sobre semilla de trigo con el fin de favorecer la sacarificación final.

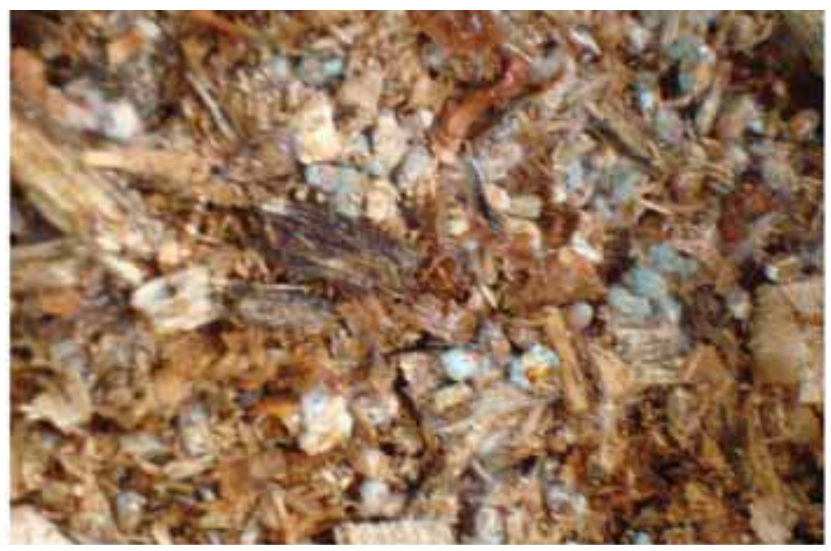

Figura 1. Crecimiento fúngico en el tratamiento de madera.

\section{Seguimiento de la FSS}

Para evaluar el tratamiento a escala banco se tomaron muestras en diferentes días y se realizaron las siguientes determinaciones:

\section{Porcentaje de humedad, mediante secado a $65^{\circ} \mathrm{C}$ hasta} lograr peso constante.

Para este ensayo, de las cinco bandejas tratadas en las mismas condiciones se seleccionaron al azar la número 2 y la número 5 , en tanto la número 1 actúa como blanco. Para la toma de muestra, de cada bandeja se realizó una mezcla de submuestras tomadas en las cuatro esquinas y en el centro de cada una.

2. FDA (Fibra detergente ácida). PEC QCO MPCC F 003 (*) FDN (Fibra detergente neutra). PEC QCO MOCC F 004 (*) Lignina. PEC QCO MPCC F 003 (*)

Para este ensayo, de las cinco bandejas tratadas en las mismas condiciones se seleccionaron al azar la número 3 y la número 6 , en tanto la número 1 actúa como blanco. Para la toma de muestra, de cada bandeja se realizó una mezcla de submuestras tomadas en las cuatro esquinas y en el centro de cada una.

Las muestras fueron secadas y molidas de manera de obtener un granulado homogéneo que permitiera obtener resultados comparables en los duplicados realizados para cada muestra, no superando $1 \%$ de diferencia entre los resultados obtenidos.

Para ello se procedió a secar $100 \mathrm{~g}$ de cada muestra en estufa a $65^{\circ} \mathrm{C}$ hasta peso constante y luego se molió en un molino martillo.

\section{Análisis de azúcares liberados luego de la sacarificación} enzimática de residuos de madera con y sin tratamiento. (*)

Se extrajeron al azar muestras de las bandejas número 1,2 y 3 del borde y centro de cada bandeja, rotulándose 1, 2 y 3 a las extraídas del borde de la bandeja (mezcla de las cuatro esquinas) y 1', 2' y 3' a las extraídas del centro. Se secaron las muestras en estufa a $65^{\circ} \mathrm{C}$ hasta peso constante. Se pesaron $2 \mathrm{~g}$ de muestra seca en un erlenmeyer de $125 \mathrm{ml}$, se agregaron $25 \mathrm{ml}$ de buffer acetato $\mathrm{pH} 4.8$ y $5 \mathrm{ml}$ de una solución de enzima celulasa (solución con $20 \mathrm{~g} / 1$ de enzima con $40.000 \mathrm{u} / \mathrm{g}$ ), lo que corresponde a $4000 \mathrm{u}$ de enzima.

Además, para verificar la actividad enzimática en otro erlenmeyer, rotulado "papel", se pesaron $2 \mathrm{~g}$ de papel de filtro whatman $\mathrm{N}^{\circ} 1$ con $25 \mathrm{ml}$ de buffer acetato y $5 \mathrm{ml}$ de la solución enzimática. Todos los erlenmeyers se mantuvieron en baño de agua sin agitación durante 6 hs. a $50{ }^{\circ} \mathrm{C}$ y luego se retiraron los recipientes a freezer para analizar al día siguiente su contenido en azúcares reductores por el método de DNS.

Método de DNS: Se colocaron 0,4 $\mathrm{ml}$ del filtrado en un tubo de ensayo y se agregaron $4 \mathrm{ml}$ de reactivo DNS. Paralelamente se realizó un blanco con $0,4 \mathrm{ml}$ de agua destilada.

Se incubó a $100{ }^{\circ} \mathrm{C}$ durante 10 minutos y se midió absorbancia relativa al blanco a $540 \mathrm{~nm}$. Se realizó una curva de calibración utilizando soluciones de glucosa de $0,8,1,6,3,2,4,8$ y $6,4 \mathrm{mg} / \mathrm{ml}$, las cuales se prepararon a partir de una solución estándar de $8 \mathrm{mg} / \mathrm{ml}$.

(*) Para poder realizar estos ensayos las muestras fueron secadas y molidas de manera de obtener un granulado homogéneo. Para ello se procedió a secar $100 \mathrm{~g}$ de cada muestra en una estufa a $65^{\circ} \mathrm{C}$ hasta obtener peso constante y luego se molió en un molino martillo.

Para evaluar los resultados que podrían obtenerse con diferentes concentraciones de enzima, se realizaron hidrólisis sobre la muestra rotulada 2'. Para esto se secó una muestra a $80^{\circ} \mathrm{C}$ hasta peso constante y se molió para conseguir tamaño homogéneo de partículas. Se colocaron $5 \mathrm{~g}$ del material molido y $50 \mathrm{~mL}$ de agua destilada. El pH se ajustó a 4,8 con solución de $\mathrm{HCl} 10 \%$ (v/v). El tratamiento enzimático se efectuó durante 6 hs. a $45^{\circ} \mathrm{C}$ en baño de agua con agitación. Se trabajó con cuatro concentraciones de enzima diferentes: $80 \mathrm{u} / \mathrm{ml}$, $120 \mathrm{u} / \mathrm{ml}, 160 \mathrm{u} / \mathrm{ml}$ y $200 \mathrm{u} / \mathrm{ml}$ para los $5 \mathrm{~g}$ de sustrato. Se realizaron duplicados para cada concentración ensayada.

\section{Identificación de productos de degradación de lignina.}

Se retiraron muestras de las seis bandejas. Para la toma de muestra, de cada bandeja se realizó una mezcla de submuestras tomadas en las cuatro esquinas y en el centro de cada una. A éstas se le agregó una solución de $\mathrm{NaOH}$ al $30 \%$ en una relación de $6 \%(\mathrm{p} / \mathrm{v})$. Se mantuvieron durante 30 minutos a $30{ }^{\circ} \mathrm{C}$ con agitación intermitente.

Para cada una de ellas se determinaron las absorbancias a longitud de onda $254,280,310$ y $354 \mathrm{~nm}$.

\section{Determinación de azúcares reductores a partir del proceso} realizado a escala de $57 \mathrm{~K}$ (campo).

Para evaluar una primera aproximación de los resultados obtenidos para un pequeño aumento de escala, se realizó la determinación de azúcares reductores a partir del proceso realizado a escala de 57 $\mathrm{K}$ (campo). Para ello se extrajeron muestras al azar de dos cajones ensayados. En ambos se retiró una muestra un mes después de la inoculación con Tramentes versicolor y Pleurotus ostreatus (1 y 1A) y una muestra luego de la inoculación con Trichoderma reesei (2 y 2A). Se secaron las muestras en estufa a $65^{\circ} \mathrm{C}$ hasta peso constante. Se trabajó con una concentración de enzima de $10.000 \mathrm{u}$.

\section{Resultados}

Los resultados se exponen en el mismo orden en el que fueron presentados los procedimientos en la sección Materiales y Métodos.

1. Los resultados correspondientes a la determinación del porcentaje de humedad se exponen en la Tabla 1. La humedad se mantuvo en un rango entre 55 y $65^{\circ} \mathrm{C}$ durante todo el proceso, lo cual es adecuado para los requerimientos de los hongos empleados. 


\begin{tabular}{|l|c|c|}
\hline Fecha de extracción & $\mathbf{N}^{\mathbf{0}}$ de bandeja & \% Humedad \\
\hline \multirow{3}{*}{ Día I } & 1 & 59,8 \\
\cline { 2 - 3 } & 2 & 57 \\
\hline \multirow{3}{*}{ Dia 30 } & 5 & 58,9 \\
\cline { 2 - 3 } & 1 & 65,3 \\
\hline Día 70 & 2 & 66,3 \\
& 5 & 63,8 \\
\hline \multirow{2}{*}{ Día 85 } & 1 & 58 \\
& 2 & 55 \\
& 5 & 59,6 \\
\hline
\end{tabular}

Tabla 1. Determinación del \% de humedad.

2. Los resultados correspondientes a FDA, FDN y lignina se expresan en la Tabla 2.

\begin{tabular}{|c|c|c|c|c|}
\hline & $\begin{array}{l}\text { Bandeja I } \\
\text { (miestra def dia in }\end{array}$ & $\begin{array}{c}\text { Bandeja } 1 \\
\text { (miestra def dia as? }\end{array}$ & $\begin{array}{l}\text { Bandeja } 3 \\
\text { timpedan del dia } 85\end{array}$ & $\begin{array}{c}\text { Bandeja } 6 \\
\text { meara det dias } 85\end{array}$ \\
\hline $\begin{array}{l}\text { Lignina } \\
\text { (g/100g) }\end{array}$ & 42 & 43 & 38 & 41 \\
\hline $\begin{array}{l}\text { FDA } \\
\text { Fibra detergente } \\
\text { ácida (g/100g) }\end{array}$ & 70 & 69 & 66 & 55 \\
\hline $\begin{array}{l}\text { FDN } \\
\text { Fibra detergente } \\
\text { neutra }(\mathrm{g} / 100 \mathrm{~g})\end{array}$ & 81 & 83 & 72 & 74 \\
\hline
\end{tabular}

Tabla 2. Determinación de lignina, FDA y FDN.

En los resultados no se observaron cambios significativos en el parámetro correspondiente a lignina. Debido a que podría inferirse una tendencia a disminuir, se evaluaron los productos de degradación de lignina mediante la medida de absorbancias a longitudes de onda entre 200 y $400 \mathrm{~nm}$ (ver punto 6).

En el caso de FDA se observa una tendencia más clara a la disminución respecto al blanco, al igual que en la modificación en fibras totales FDN incluida la celulosa (posiblemente hidrolizada de forma parcial).

3. Análisis de azúcares liberados luego de la sacarificación enzimática de residuos de madera con y sin tratamiento. Los resultados de azúcares reductores obtenidos luego de la sacarificación enzimática se señalan en la Tabla 3.

\begin{tabular}{|l|c|c|c|}
\hline Muestra & Absorbancia sou & $\begin{array}{c}\text { Concentración } \\
\text { de glucosa }(\mathrm{g} / \mathrm{l})\end{array}$ & Promedio \\
\hline 1 & 0,1797 & 1,516 & 1,27 \\
\hline $\mathrm{I}$ & 0,1119 & 1,032 & \\
\hline 2 & 0,2489 & 2,011 & - \\
\hline $2^{\prime}$ & 0,0970 & - & \\
\hline 3 & 0,2492 & 2,013 & 1,86 \\
\hline $3 \cdot$ & 0,1851 & 1,555 & \\
\hline Papel & 1,1647 & 8,559 & \\
\hline
\end{tabular}

Tabla 3. Azúcares reductores obtenidos luego de la sacarificación enzimática (la muestra Papel corresponde a un standard).

Ecuación de la recta de regresión de la curva de calibración con glucosa:
$\mathrm{A}_{570}=0,13987$ [Glucosa] - 0,03240
$\mathrm{R}^{2}=0,99872$

[Glucosa $]_{\mathrm{B}}=(0,1458+0,03240) / 0,13987=1,2740 \mathrm{~g} / \mathrm{L}$

$[\text { Glucosa }]_{\mathrm{T}}=(0,2277+0,03240) / 0,13987=1,8596 \mathrm{~g} / \mathrm{L}$

Pudo apreciarse que el rendimiento del tratamiento se encuentra por encima del logrado sin tratamiento con hongos:

Rendimiento $(\%)=1,8596 \times 100 / 1,2740=146 \%$

Este resultado indica que podría conseguirse un $46 \%$ más de liberación de azúcares mediante el tratamiento de la madera con estos hongos.

Los resultados obtenidos en la hidrólisis con mayores concentraciones de enzima sobre la muestra rotulada 2 ', se expresan en la Tabla 4. Para esto se secó una muestra a $80{ }^{\circ} \mathrm{C}$ hasta peso constante y se molió para conseguir tamaño homogéneo de partículas. Se colocaron $5 \mathrm{~g}$ del material molido y $50 \mathrm{~mL}$ de agua destilada. $\mathrm{El} \mathrm{pH}$ se ajustó a 4,8 con solución de $\mathrm{HCl} 10 \%(\mathrm{v} / \mathrm{v})$. El tratamiento enzimático se efectuó durante 6 hs. a $45^{\circ} \mathrm{C}$ en baño de agua con agitación.

\begin{tabular}{|l|c|c|c|}
\hline Muestra & Unidades enzima & A ss & Glucosa (g/) \\
\hline IA & 4000 & 0,42 & 3,2 \\
\hline 2 & 4000 & 0,42 & 3,2 \\
\hline $2 \mathrm{~A}$ & 6000 & 0,53 & 4 \\
\hline 3 & 6000 & 0,49 & 3,7 \\
\hline $3 \mathrm{~A}$ & 8000 & 0,47 & 3,6 \\
\hline 4 & 8000 & 0,55 & 4,2 \\
\hline $4 \mathrm{~A}$ & 10000 & 0,59 & 4,4 \\
\hline
\end{tabular}

Tabla 4. Hidrólisis enzimática del sustrato.

Se observa una tendencia al aumento de la glucosa liberada con la concentración de enzima. También se aprecia variabilidad en los duplicados, probablemente inherente a la variabilidad del sustrato. Esto nos indica que deben optimizarse las condiciones de la hidrólisis para lograr los mejores resultados.

4. Determinación de productos de la degradación de lignina. Los resultados obtenidos están contenidos en la Tabla 5.

\begin{tabular}{|c|c|c|c|c|}
\hline MUESTRA & i.354 & 2.310 & 2.280 & .254 \\
\hline Bandeja 1 & 0,2303 & 0,3744 & 0,5426 & 0,6662 \\
\hline Bandeja 1 iantua & 0,2165 & 0,3551 & 0,5135 & 0,6314 \\
\hline Bandeja 2 & 0.4066 & 0,6685 & 0,9502 & 1,1831 \\
\hline Bandcja 3 & 0,4132 & 0,6785 & 0,9506 & 1,1837 \\
\hline Bandeja 4 & 0,3024 & 0,5045 & 0,7247 & 0,9056 \\
\hline Bandeja 5 & 0,4701 & 0,7713 & 1,0977 & 1,3620 \\
\hline Bandeja 6 & 0,3456 & 0,5743 & 0,8076 & 0,9996 \\
\hline
\end{tabular}

Tabla 5. Absorbancias específicas de productos de degradación de lignina.

De los espectros realizados se ve un incremento en las absorbancias a 354, 310, 280, $254 \mathrm{~nm}$ de las muestras con tratamiento. Este aumento puede estar asociado al incremento en las mismas de compuestos monoméricos u oligoméricos de lignina degradada. Asimismo, puede asociarse con procesos oxidativos de la lignina, lo cual permitiría obtener mayor cantidad de estos compuestos.

5. Los resultados de la determinación de azúcares reductores a partir del proceso realizado a escala de $57 \mathrm{~K}$ (campo) se muestran en la Tabla 6. Los azúcares obtenidos en las muestras 1 y 2 son muy bajos, pero aumentan claramente en las muestras $1 \mathrm{~A}$ y $2 \mathrm{~A}$. Este aumento podría deberse a que éstas ya estaban pretratadas, tanto para lograr una deslignificación (Tramentes versicolor y Pleurotus ostreatus) como una presacarificación (Trichoderma reesei). Este resultado hace pensar también en la posibilidad de aumentar la escala del proceso sin mayores dificultades. 


\begin{tabular}{|l|c|c|}
\hline $\begin{array}{l}\text { Identificación } \\
\text { de la muestra }\end{array}$ & Absorbancia & [Glucosa| (g/) \\
\hline 1 & 0,2056 & 0,49 \\
\hline 2 & 0,2348 & 0,55 \\
\hline IA & 2,1344 & 4,20 \\
\hline 2A & 3,2860 & 6,42 \\
\hline
\end{tabular}

Tabla 6. Azúcares reductores obtenidos luego de la sacarificación enzimática (escala campo).

\section{Conclusión}

Es posible concluir que los residuos forestales evaluados en este trabajo son utilizados como sustrato y modificados por los hongos evaluados.

En general, las metodologías de hidrólisis de lignocelulósicos a azúcares fermentables utilizadas son químicas o enzimáticas y requieren instalaciones de planta con inversiones costosas.

El pretratamiento diseñado implica una manipulación simple, lo cual permite un escalado a nivel productivo sin grandes inversiones. Esta propuesta permitiría obtener oligómeros de celulosa por una presacarificación en fermentación semisólida.

Los resultados alcanzados hacen posible considerar que la optimización de este proceso podría mejorar los rendimientos, con un aumento en los azúcares fermentables obtenidos.

El costo del pretratamiento es bajo y puede emplearse con un doble propósito simultáneo: tratamiento de mejora de proceso y gestión del residuo.

El sistema de fermentación sólida permite realizar un diseño de tratamiento durante el estoqueo, mediante el cual se inocula el material disponiéndolo en trincheras.

En el rendimiento a escala piloto, el resultado primario del aumento del $46 \%$ en azúcares reductores podría indicar que de aplicarse este tratamiento durante un tiempo más prolongado se alcanzarían mejores rendimientos y una mayor transformación.

La conversión de estos azúcares a alcohol por las tecnologías tradicionales es ya bien conocida, al igual que sus rendimientos.

La optimización de esta metodología permitirá su utilización, contribuyendo con el objetivo de lograr una mayor transformación de la biomasa lignocelulósica. Se deberá seguir trabajando en la conformación consorcial de los agentes (hongos) y en los procesos de extracción de los azúcares y formulación de una melaza comercial a partir de madera. Estos son algunos de los nuevos desafíos a considerar para trabajos futuros.

\section{Reconocimientos}

Este trabajo se realizó en conjunto con la Empresa NALAVIP S.A. (Grupo de Industrias de la madera del Departamento de Paysandú) y con la Intendencia Municipal de Paysandú quienes colaboraron activamente en el desarrollo de este proyecto.

\section{Referencias}

- ATTFIELD, P.; BELL P. Use of population genetics to derive nonrecombinant Saccharomyces cerevisiae strains that grow using xylose as a sole carbon source. En: FEMS Yeast Research. 2006 (6/6):862-868.

- BHAT, M. Research review paper: cellulases and related enzymes in biotechnology. En: Biotechnology Advances. 2000, (18):355383.

- CANEL, E.; MOO-YOUNG, M. Solid state fermentation systems. En: Process Biochemistry. 1980, (15):24-28.

- CHAHAL, D. Bioconversions of poysaccharides of lignocellulose and simultaneous degradation of lignin. En: KENNEDY et al. Lignocellulosics: science, technology, development and use.
London: Ellis Horwood Limited, 1992. pp. 83-93.

- CHAHAL, P.; CHAHAL, D. Production of cellulose in solid state fermentation with Trichoderma reesei MCG 80 on wheat straw. En: Applied Biochemistry and Biotechnology. 1996, (57/58):433-442.

- CHRISTOPHERSON, C.; ANDERSON, E.; JAKOBSEN, T.; WAGNER, P. Xylanases in wheat separation. En: Starch. 1997, (49):5-12.

- COOMBS, J. EEC resources and strategies. En: Phil. Trans. R. Soc. Lond. Ser. A. 1987, (321):405-422.

- DEMAIN, A. L.; NEWCOMB, M.; WU, J.H.D. Cellulase, clostridia, and ethanol. En: Microbiology and Molecular Biology Reviews. 2005, (69):124-154.

- GOLD, M.H.; ALIC, M. Molecular bilogy of the lignin-degrading basidiomycete Phanerochaete chrysosporium. En: Microbiology and Molecular Biology Reviews. 1993, 57(3):605-622.

- HALTRICH, D.; NIDETZKY, B.; KULBE, K.D., et al. Production of fungal xylanases. En: Bioresource Technology. 1996, (58):137161.

- JECU, L. Solid-state fermentation of agricultural wastes for endoglucanase production. En: Industrial Crops and Products. 2000, (11):1-5.

- KRAUSE, D.O. Opportunities to improve fiber degradation in the rumen. En: FEMS Microbiology Reviews. 2003, (27):663-393.

- LAWFORD, H.G.; ROUSSEAU, J.D. Steady-state measurements of lactic acid production in a wild-type and a putative D-lactic acid dehydrogenase-negative mutant of Zymomonas mobilis: influence of glycolytic flux. En: Applied Biochemistry and Biotechnology. 2002, (429):98-100.

- LEONOWICZ, A. Cooperation of fungal laccase and glucose 1-oxidase in transformation of björkman lignin and some phenolic compounds. En: Holzforschung. 1999, (53):376-380.

- LEVINE, J. S. Biomass burning and global change. En: LEVINE, J. S. Remote sensing and inventory development and biomass burning in Africa. v.1. Massachusetts: MIT Press, 1996. pp.35

- MALHERBE, S.; CLOETE, T.E. Lignocellulose biodegradation: fundamentals and applications. En: Reviews in Environmental Science and Biotechnology. 2003, (1):105-114.

- MOSIER, N. et al. Features of promising technologies for pretreatment of lignocellulosic biomass. En: Bioresource Technology. 2005, 96(6):673-686.

- MOSIER, N. Dry grind processing bridge to cellulosic ethanol. ag. and bio. engineering [En línea]. West Lafayette: Purdue University, s.d. [Consulta: enero 2006]. Disponible en: fairway.ecn.purdue.edu/ lorre/16/research/bioenergy_symposiummosier-22FEB06.pdf

- NGUYEN, Q. A. Economic analyses of integrating a biomassto-ethanol plant into a pulp/saw mill. En: SADDLER, et al. Bioconversion of forest and agricultural plant. s.1.: CAB International, 1993. pp. 321-340.

- RAGAUSKAS, A.J. The path forward for biofuels and biomaterials. En: Science. 2006, (311):484-489.

- RAJARTHANAM, S. Biodegradation and biosynthesis capacities of mushrooms: present and future strategies. En: Critical Review in Biotechnology. 1998, 18(283):91-236.

- SMITH, J. E.; ANDERSON, J. G.; SENIOR, E.K., et al. Bioprocessing of lignocelluloses. En: Phil. Trans. R. Soc. Lond. Ser. A. 1987, (321):507-521.

- SUN, Y.; CHENG, J. Hydrolysis of Lignocellulosic Materials for Ethanol Production: A Review. En: Bioresource Technology. 2002, 83(1):1-11.

- ZHANG, Y.H.; LYND, L.R. Cellulose utilization by Clostridium thermocellum: Bioenergetics and hydrolysis product assimilation. En: Proceedings of the National Academy of Sciences. 2005, (102):7321-7325. 